\title{
New possibilities of worm thread machining and practice of implementation
}

\author{
Evgeniy Trubachev* and Kirill Bogdanov \\ Institute of Mechanics named after Prof. V.I. Goldfarb, Kalashnikov ISTU, 426069 Izhevsk, Studencheskaya str. 7, Russia
}

\begin{abstract}
Thread grinding is the traditional operation of final machining for worms and other critical threads. However, similar to other types of tooth grinding, this type of machining is expensive; it requires application of high cost equipment and needs valuable workers. In many practical cases, its alternative can be the application of edge machining, especially as the possibilities of controlling the corresponding advanced machine-tools and their accuracy have been increased significantly. This paper describes the experience of implementation of edge machining of worm threads within a low-series production of spiroid and worm gearboxes for pipeline valves gained at the Institute of Mechanics named after prof. V.I. Goldfarb of Kalashnikov Izhevsk State Technical University and at the Small Innovative Enterprise "Mechanik". Schemes of thread machining by straight and radial parts of a single cutter and by a face cutter head are considered. The technique is implemented, and it provides the reduction of labor intensity and prime cost of both the preliminary and final machining of worms, including heat-strengthened ones; and it also results in the correctly localized contact in worm and spiroid gears.
\end{abstract}

\section{Introduction}

Machining operations for teeth and turns of threads are strongly determining the labor intensity and quality of machines, that is why, improvement of tooth machining is traditionally an urgent issue [1]. As soon as turns of critical and loaded threads and worms are preliminary slotted, they are usually subjected to heat treatment and further grinding. Grinding operations are performed at the high precision and expensive equipment provided with all necessary devices for fine correction of parameters - height, thickness, angles and radii of thread profiles. One of the hardest organizational problems for Russian manufacturers is the selection and retention of employees capable of performing these operations. Preparation of a high-skilled thread grinder is a lasting task that is not always possible for an enterprise. At the same time, the employment market provides a sufficient (and even increasing) number of operators for $\mathrm{CNC}$ machine-tools (lathes, grinding and boring ones and other) and even centers of their training. Moreover, it is common that possibilities of thread and worm grinding machine-tools are abundant for machined worms and threads (even rather critical, though non-precision ones); therefore, it becomes clear that it is exciting for a manufacturer to master machining of threads (including heat-strengthened ones up to HRC $50 \ldots 60$ ) by $\mathrm{CNC}$ thread cutting lathes. There is a successful practice of machining surfaces as well as tooth surfaces by hardalloy tools [2, 3, 4], but the limiting factor for finishing edge machining of threads is the absence of fine adjustment for parameters of machined helical surfaces within a standard layout of these machine-tools, and rigid linkage of the obtained thread profile with the geometry of a tool and its mounting which complicates the setting and resetting of the equipment. We propose in the present manuscript several versions of solving this problem specifically for low-series diversified production, as, for example, is typical for the Small Innovative Enterprise "Mechanik" [5].

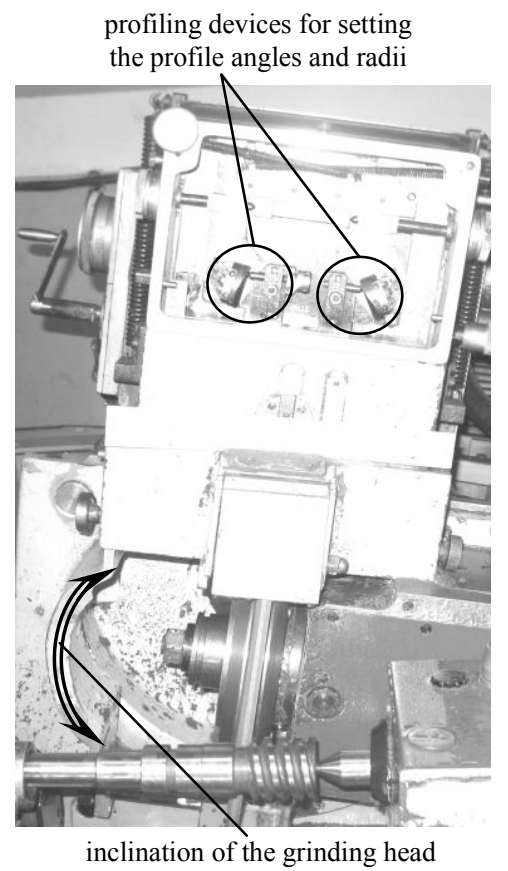

Fig. 1. Devices for resetting of the thread grinding machinetool. 


\section{Controlled thread parameters and traditional methods of control}

Let us pay attention to two important features of the existing production of critical threads:

- tolerances of the worm pitch and profile are well agreed with the accuracy parameters of the existing $\mathrm{CNC}$ thread cutting lathes (for instance, for the $8^{\text {th }}$ degree of worm accuracy in accordance with the State Standard ГОСТ $3675-81$ and the $5^{\text {th }}$ grade of thread accuracy, the tolerances are within $\pm 19 \ldots \pm 53 \mathrm{mcm}$ );

- the important existing requirement to the thread profile is to provide its curvature (radius of curvature) or, moreover, its correspondence to the assigned set of points; it allows for providing the necessary localization of the initial adjacency (contact pattern) of threads with the coupled surfaces and, correspondingly, to achieve the reduced sensitivity of couplings to inevitable errors and strains and the increased load carrying characteristics of couplings.

The traditional approach [6] consists fundamentally in the selection of such parameters for the thread machine-tool settings (parameters of tool geometry, its setting and motion) that will provide the assigned thread profile and pitch. Because of the action of a great number of manufacturing factors, the actually obtained thread geometry is a certain approximation to the calculated one; that is why, the resetting of equipment is usually required. This situation is easily and quickly corrected at grinding by profile dressing of the wheel and slight variation of its position. Thread grinding machine-tools are normally equipped with the corresponding devices (Fig. 1) that are controlled mechanically or in software.

Dressing and variation of the edge tool position are more complicated. If the manufacturer wants to increase the efficiency of heat strengthened thread machining and applies the tool with multifaceted throw-away inserts that have rectilinear main cutting edges in most cases, this dressing is hardly justified in real production. Several versions of relatively simple solutions to overcome the pointed difficulty are considered further.

\section{Thread profile is generated by a rectilinear cutting edge of the cutter}

This is practically the classical version of thread generation for ZN2 (SZN2) worms [7] - Fig. 2. It appears that the only significant difference here is that the front plane of the cutter can be shifted by the value $y_{0}$ relative to the worm centre line.

Coordinates of points of the axial profile formed by the generating line can be determined in accordance with the following sequence:

- to assign points ( $i$ is the number of each point) on the generating straight line within the height of the cutter profile; it is convenient to use the coordinate system (CS) $S_{0}\left(x_{0}, y_{0}, z_{0}\right)$ :

$$
z_{0 i}= \pm\left[\frac{s_{0}}{2}+\left(x_{0 i}-\frac{d_{1}}{2}\right) \tan \alpha_{0}\right]
$$

- to determine coordinates of these points in $\operatorname{CS}(x, y, z)$ :

$$
\begin{aligned}
& x_{i}=x_{0_{i}}, \\
& y_{i}=y_{0} \cos \gamma_{0}-z_{0 i} \sin \gamma_{0}, \\
& z_{i}=y_{0} \sin \gamma_{0}+z_{0 i} \cos \gamma_{0},
\end{aligned}
$$

where $\gamma_{0}$ is the angle of the cutter front plane inclination relative to the centre line (it is usually chosen to be equal or close to the reference helix angle of the worm / the average helix angle of the thread);

- to normalize points to the plane of the axial section $x O z=r O z(r)$ :

$$
\begin{aligned}
& r_{i}=\sqrt{x_{i}^{2}+y_{i}^{2}} \\
& z(r)=z_{i}-p_{\gamma} \arctan \left(y_{i} / x_{i}\right),
\end{aligned}
$$

where $p_{\gamma}$ is the helix parameter of the generated surface.

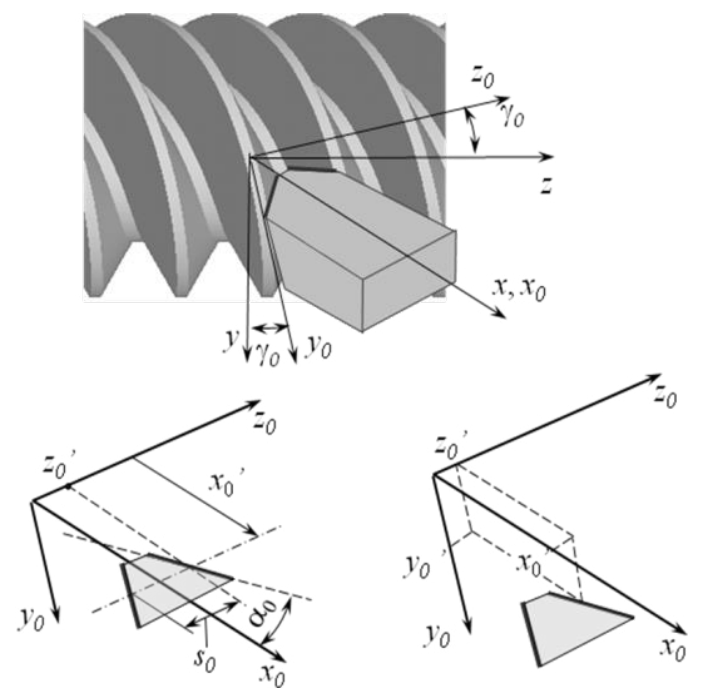

Fig. 2. Scheme of machining by a cutter and applied coordinate systems.

The shift $y_{0}$ of the cutter that can be easily recalculated within the setting into the corresponding shift in machine-tool coordinates as $\gamma=\gamma_{0} \cos \gamma_{0}$ influences the geometry of the generated thread. Fig. 3a shows the variation of the axial profile of the slotted inter-turn root of the four-threaded spiroid worm with the axial profile $m_{x}=3.35 \mathrm{~mm}$ and profile angles of the right and left thread flanks $12^{\circ}$ and $28^{\circ}$, correspondingly. For better visual perception, the deviations from the profile obtained by the non-shifted cutter are zoomed by 10 times in this Figure. It is evident, that the shift of the cutter downwards the centre line causes a slight cut-off of the thread dedendum for right thread flanks and the addendum for left ones, while the shift upwards causes the opposite effect. Provided that a slight correcting shift $\Delta z$ of the cutter is introduced along the axis $z$, there is a 
way that the part of the thread profile will represent the combination of three or more segments generated at various shifts of $y_{0}-$ see Fig. $3 b$.

Therefore, the main idea of this scheme of thread machining is to use several cutters mounted in the turret head; and each of these cutters is generating its section of the thread profile. In order to form each of two opposite thread flanks, it is convenient to apply an individual group of cutters; and the cutters in groups can be the same differing only by their mounting relative to the centre line. The number of cutter passes and machining time are insignificantly increased, and the main "cost" of the obtained possibility of controlling the thread profile geometry is the necessity to use several cutters.

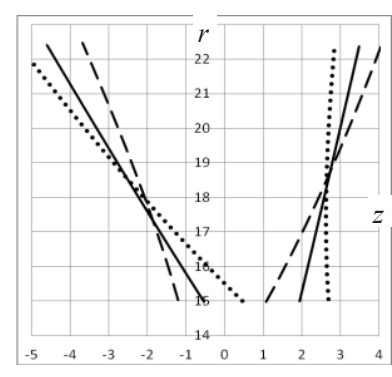

a)

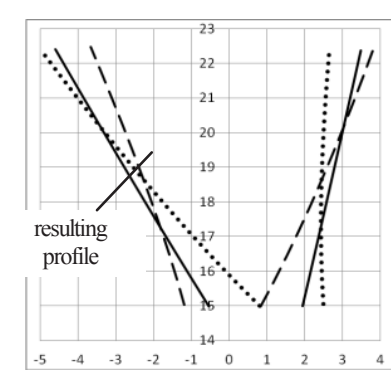

b)
Fig. 3. Axial profiles at the cutter shift: --0 (non-shifted cutter); - - downwards by $1.0 \mathrm{~mm}, \cdots$ - upwards by $1.0 \mathrm{~mm}$

This scheme can also be implemented at the threeaxis (axes " $X$ ", " $Y$ ", " $Z$ ") lathe machining center; in this case, one cutter is sufficient, and its arrangement relative to the center line can be varied by means of the control software during machining.

The resulting profile shown in Fig. $3 b$ by a heavy gray line turns to be slightly broken, the discontinuity of transition between segments can be quantitatively evaluated by the difference $\Delta \mathrm{a}_{\mathrm{x}}$ in profile angles in the area of segments coupling. It can be decreased at the increase of the number of coupling segments, and depending on the method implementation it leads to the increase either in the number of tools applied at setting and their operating strokes, or only in the number of operating strokes.

Another way of controlling the profile geometry is based on the feature of ZN2 (SZN2) worms substantiated in [8], that the axial profile of the worm turns to be concave for the traditional cutting scheme (for the cutter which is not shifted relative to the centre line). Thus, the radii of concave profiles for the considered example are about 1160 and $280 \mathrm{~mm}$ correspondingly for the right and left flanks. However, if the initial arrangement of the cutter prior to the proposed shift is already higher or lower than the centers, the profile curvature is changed (in opposite directions for opposite flanks, as expected); and the profile can become convex, thus smoothing the coupling of segments and allowing to change the profile slope (Table 1).

\section{Thread profile is generated by a circular cutting edge of the cutter}

This version implies that in order to generate the final thread profile, one should apply the cutter with a throwaway rhombic insert with a big radius roundness (Fig. 4a). The final thread profile is generated by a multifold of positions of the generating circumference - a circular part of the cutting edge - which is displacing along the worm thread profile.

Table 1. Parameters of thread profile modification at tool shift by $1 \mathrm{~mm}$.

\begin{tabular}{|c|c|c|c|}
\hline $\begin{array}{c}\text { Average } \\
\text { shift } \boldsymbol{y}_{\mathbf{0 a v}} \text { of } \\
\text { the cutter }\end{array}$ & $\begin{array}{c}\text { Generated } \\
\text { surface }\end{array}$ & $\begin{array}{c}\text { Profile slope } \\
\text { at the thread } \\
\text { apex/root, } \\
\mathbf{m c m}\end{array}$ & $\begin{array}{c}\text { Difference } \\
\Delta \boldsymbol{\alpha}_{\boldsymbol{x}} \text { at } \\
\text { points of } \\
\text { coupling at } \\
\text { the thread } \\
\text { apex/root, }\end{array}$ \\
\hline $\begin{array}{c}0 \mathrm{~mm} \\
\text { (non-shifted } \\
\text { cutter) }\end{array}$ & Light & $27 / 47$ & $0.85 / 1.12$ \\
\hline$-2 \mathrm{~mm}$ & Right & $12 / 23$ & $0.82 / 1.04$ \\
\hline$+3 \mathrm{~mm}$ & Left & $45 / 70$ & $0.80 / 1.05$ \\
\hline & & & $0.67 / 0.84$ \\
\hline
\end{tabular}

The front plane of the cutting tool is inclined relative to the centre line at an angle $\gamma_{0}$; that is why, the arc $\mathrm{AB}$ of the generating circumference participating in the right flank formation is located above the centre line (Fig. 4b), and the arc CD participating in the left flank formation is below the centre line (for the right direction of threads) (Fig. 4c). The final covering surface [9] of the multifold of cutting tool positions lies in the plane shifted relative to the centre line by the distance $-y_{0 p}$ when machining the right flank and $+y_{0 p}$ when machining the left one.

Points of profiles lying in these planes can be determined by the formula:

$$
\begin{aligned}
x_{\Delta i} & =\sqrt{x_{i}^{2}-\Delta y_{i}^{2}}, \\
z_{\Delta i} & =z_{i}-p_{\gamma} \arcsin \left(\Delta y_{i} / x_{i}\right),
\end{aligned}
$$

In order to apply standard replaceable hard-alloy cutting inserts within implementation of this scheme of thread profile formation, the gear design process should involve consideration of restrictions imposed by the geometry and dimensions of inserts. The combination of such geometric parameters of the thread profile as thickness, pitch, height, angles and radii of curvature must exclude the thread undercut.

It is evident, that the height $h$ of crests (waves of the "covering surface") depends on the number of positions of the generating circumference and, therefore, the pitch $p$ between the neighboring positions of the latter and its radius $r$ (Fig. 5). Omitting simple derivations, one can write:

$$
h=r-\sqrt{r^{2}-0.25 p^{2}}
$$




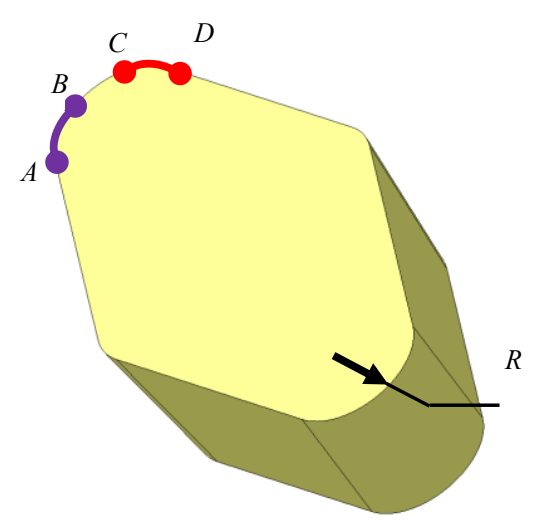

a)

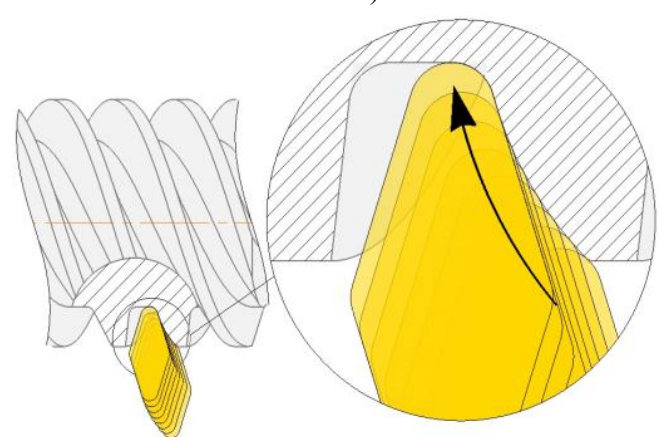

b)

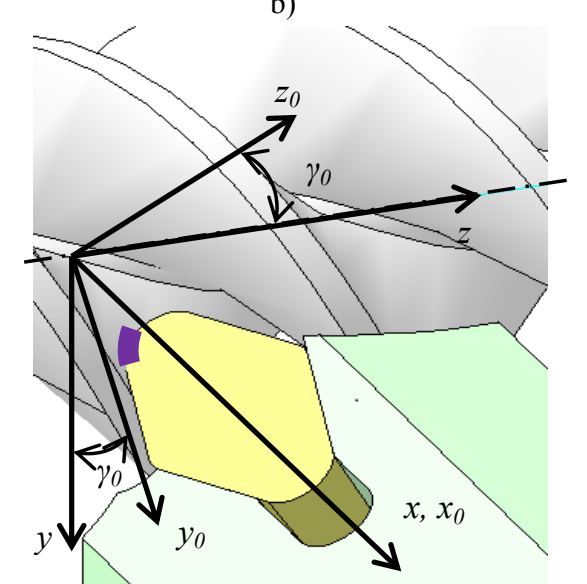

c)

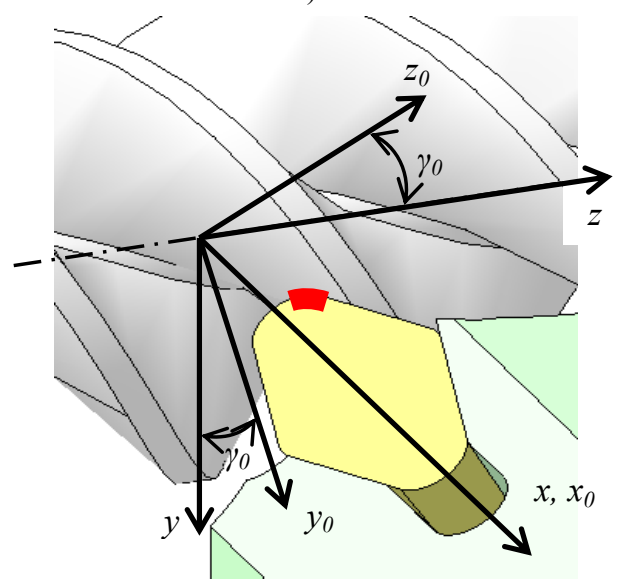

d)

Fig. 4. Insert with the circular cutting edge.

Let us give an example of machining a series of single-thread worms with the module $4 \mathrm{~mm}$. The worm profile is finally generated by 50 positions of the cutter with the rhombic insert having the radius rounding $r=$ $1.2 \mathrm{~mm}$; and it had irregularities with the height $h=5$ $\mathrm{mcm}$ in accordance with the calculation by (4). The results of measuring the thread surface roughness in circumferential direction are not more than $\mathrm{R}_{\mathrm{a}} 0.63 \mathrm{mcm}$; and measurements of the actual height of profile crests gave the result.similar to the calculation one (Figs. 6, 7).

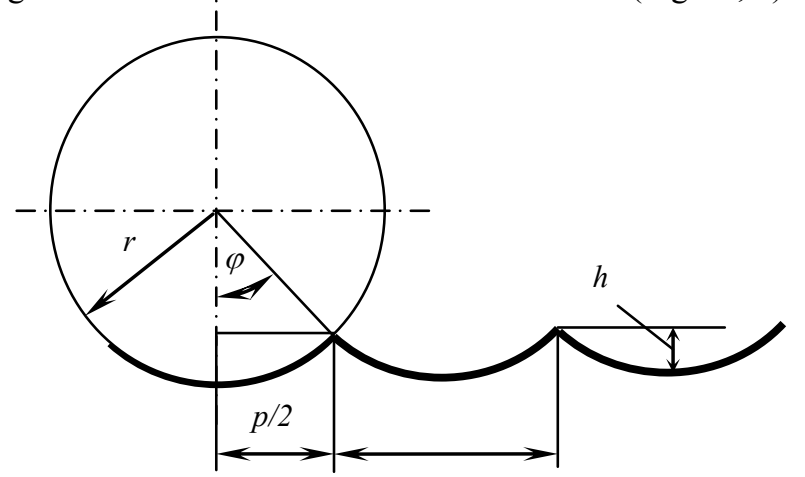

Fig. 5. Profile formation by the generating circumference - the cutting edge of the hard-allow insert

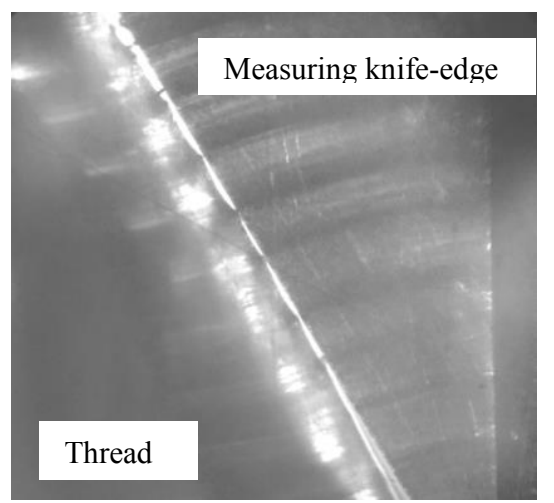

Fig. 6. Measuring of the thread surface roughness.

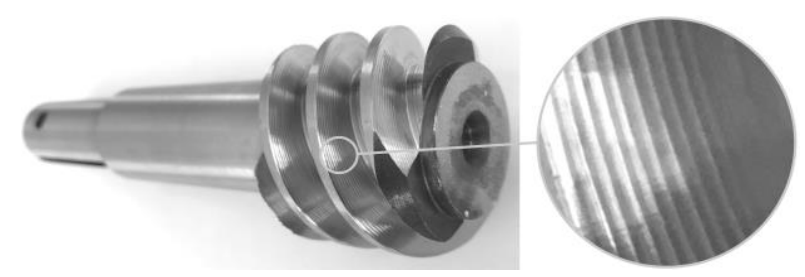

Fig. 7. Worm threads machined by a circular cutting edge of the cutter.

It should be taken into account, that such an edge machining as compared to the grinding process has the following positive aspects:

- lower inconjugacy (sharpening) of microroughness asperities [10];

- absence of indulation that appears because of disbalance and/or radial run-out of the rotating tool; - absence of the surface layer softening due to burns and there is even the naturally obtained cold hardening [11]. - special dressing of the cutting tool and additional adjusting are not required for machining of transient surfaces, curvatures in thread roots and apexes.

The time of final edge machining of worm threads was 4 minutes - twice as fast as machining at the thread grinding machine-tool. 


\section{Thread profile is generated by a face cutter head}

As for more efficient schemes of edge thread machining with application of multi-tooth tools - cutter heads - it is also possible to control the geometry relatively simply and with high accuracy, even if the tool is equipped with straight-sided hard-alloy throw-away inserts. Fig. 8 shows the scheme of thread machining by a face cutter head at the CNC thread cutting lathe [12] and the coordinate systems applied in the calculation.

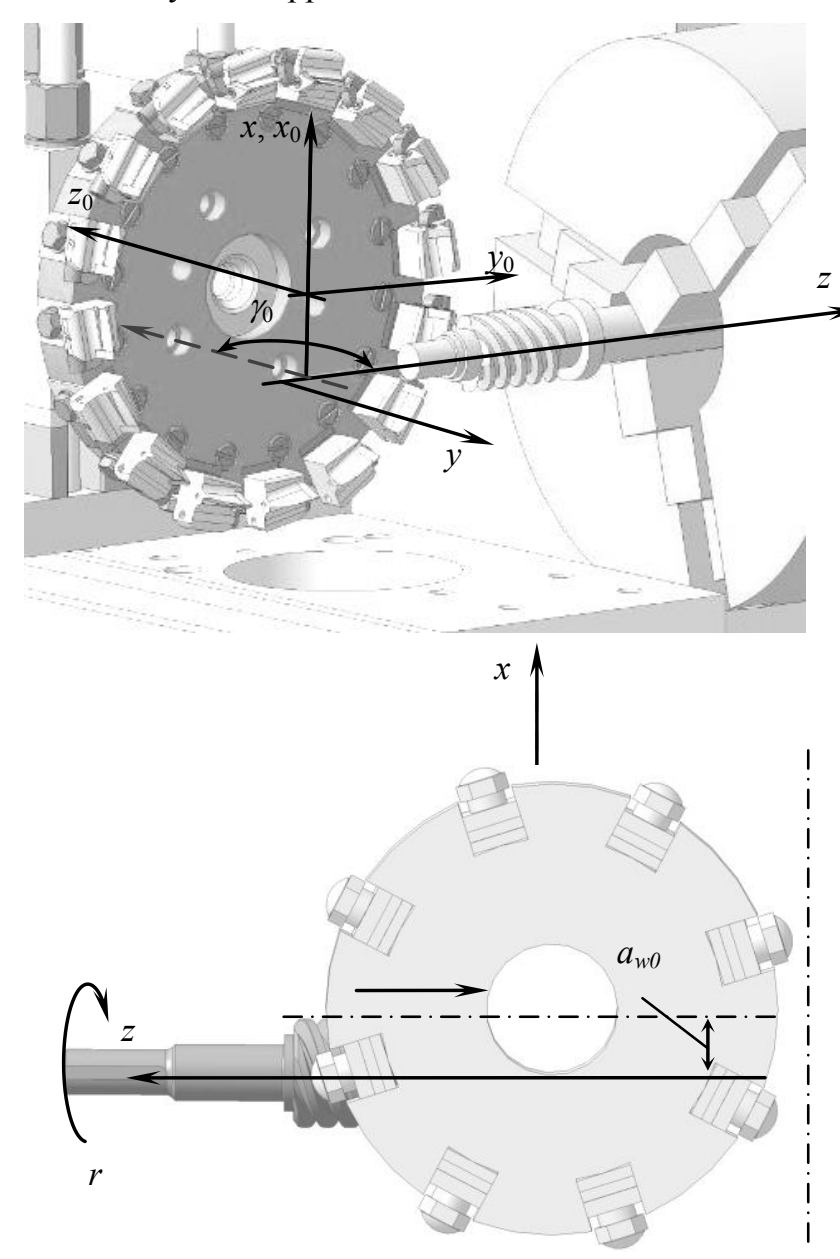

Fig. 8. Scheme of thread machining by the face cutter head at the $\mathrm{CNC}$ thread cutting lathe.

In this case, the helical surface becomes enveloping for the family of generating surfaces formed at helical relative motion (feed) of the tool. Points of the generated surface can be determined by solving the equation of meshing (contact condition at relative motion) of this surface and the generating one:

$$
\begin{aligned}
& F_{3}=\mathbf{n} \cdot \mathbf{v}_{s 0}=x_{0} z_{0} \tan \gamma_{0}+y_{0} a_{w 0}+y_{0} p_{\gamma} \tan _{\gamma 0} \mp \\
& \mp r_{0} \tan \alpha\left(x_{0}+a_{w 0}\right) \tan _{\gamma 0}-p_{\gamma 0}=0
\end{aligned}
$$

Similar to the scheme of machining by a cutter (formulas (2) and (3)), coordinates of the sought points of contact should be set to the coordinate system $S(x, y$, $z$ ) of the helical surface and further to the axial plane $x O z$.
The cutter head can be shifted a little relative to its nominal design position, and it also affects the profile geometry (Table 2). The variable parameter is the centre distance $a_{w 0}$; its design value is determined in accordance with the contact of the reference helix of the thread and the circular trajectory of cutters:

$$
a_{w 0}=0.5 d_{0} \sin \gamma_{1}
$$

where $\gamma_{I}$ is the reference helix angle of the worm, $d_{0}$ is the diameter of the cutter head. It is proposed to cut each of inter-turn roots in three steps: at the centre distance calculated by (7) (the longest pass with removal of the main part of the material) and at the introduced shift of the cutter head (two relatively short passes with removal of thin layers of the material). Advanced multicoordinate thread cutting lathes provide the shift; actually, it is the variation of the coordinate $y$ (do not confuse with the same named coordinate system $S$ ). As for simpler two-coordinate machine-tools, the shift can be made by hand at the halt of program execution.

Fig. 9 shows the profile variation for the axial section of the slotted inter-turn root of the worm with the given above parameters at the shift $\Delta a_{w 0}= \pm 1 \mathrm{~mm}$. Similar to the case of the single cutter, deviations from the nominal profile are zoomed by 10 times; and the tool is given an additional shift along the axis of the workpiece (axis $\mathrm{Oz}$ ), so that the part of the thread profile could represent the combination of three segments generated at various shifts of $\Delta a_{w 0}$.

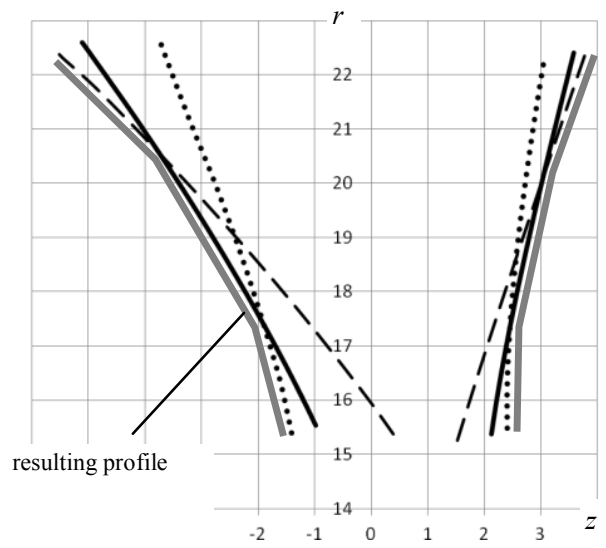

Fig. 9. Profiles generated by the face cutter head at variation of the center distance $\Delta a_{w 0}:-\Delta a_{w 0}=0 ;--\Delta a_{w 0}=1.0 \mathrm{~mm}$, $\cdots-\Delta a_{w 0}=+1.0 \mathrm{~mm}$.

It is evident, that the generation scheme allows for modifying the profile to a greater extent; firstly, because the profile is convex prior to introduction of modifying shifts.

Table 2. Parameters of profile modification at the cutter head shifting by $1 \mathrm{~mm}$.

\begin{tabular}{|c|c|c|}
\hline $\begin{array}{c}\text { Generated } \\
\text { surface }\end{array}$ & $\begin{array}{c}\text { Profile slope at the } \\
\text { thread apex/root, } \\
\text { mcm }\end{array}$ & $\begin{array}{c}\text { Difference } \Delta \boldsymbol{\alpha}_{\boldsymbol{x}} \text { at } \\
\text { points of coupling } \\
\text { at the thread } \\
\text { apex/root, }\end{array}$ \\
\hline Right & $95 / 121$ & $0.54 / 0.72$ \\
\hline Left & $262 / 256$ & $1.09 / 1.05$ \\
\hline
\end{tabular}




\section{Prospects of implementation of the new scheme of worm thread machining}

Schemes of edge thread machining proposed in the paper allow for adjusting flexibly the geometrical parameters of the worm thread profile, and first of all, the level of profile modification that provides the favorable initial contact pattern in couplings. To our opinion, the adjusting methods are rather simple; actually, they are implemented by the existing means of the common thread cutting equipment.

Note, that the following condition should be provided in order to implement the described schemes efficiently. 1. Inserts should have minimum dimension range of cutting edges. In accordance with the application experience, the deviation should not exceed $0.01 \mathrm{~mm}$. this requirement is tighter in case of applying the inserts in cutter heads; as for single cutters, the dimension range at the change of the insert can be compensated by correction of the cutter trajectory.

2. Mounting sockets for inserts in holders of cutters should have inclination (Fig. 10) that provides the close adjacency of the insert at three planes and the reliable backlash elimination at insert mounting.

3. Insert fixation should be maximum reliable that will exclude insert loosening and vibration. Our experience shows that the double fixation shown in Fig. 11 is quite suitable.

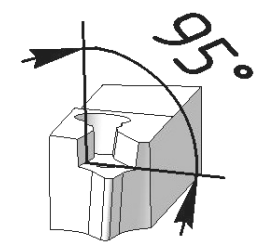

Fig. 10. Tool holder.

The said conditions do not complicate the proposed developments in any way; it is a little "cost" of new possibilities that simplify the production process.

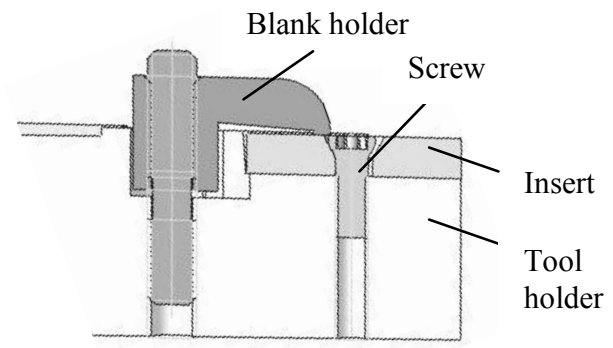

Fig. 11. Double fixation of the insert.

\section{Conclusion}

Having presented the calculation results in this paper, the authors did not imply to optimize the setting in some way; our purpose was to show the methods and efficiency of real-time controlling the geometry of threads at their machining. Issues of optimization of setting geometrical parameters and also manufacturing ones (including geometrical parameters of the tool cutting edge and parameters of operation modes), and studying the parameters of surface accuracy and quality that are important and greatly determining the efficiency of the proposed schemes are not considered in this paper and, to our opinion, they need an individual discussion. Moreover, our practice of finish machining of spiroid worm threads substantiates the exclusion of abrasive machining of threads, at least for worms having the $8^{\text {th }}$ degree of accuracy and worse.

\section{References}

1. Volkov G. Yu., Kiselev S. A., Production of gear rims with flexagons, J Vestnik ISTU, Izhevsk: ISTU, 2 (20) (in Russ.) (2017)

2. Pechenkin I. A., Puzanov V. Yu., Appilcation of 3D laser scanning for higher efficiency of machining the work surfaces of stamp tooling parts, $\mathrm{J}$ Intelligent systems in production, Izhevsk: ISTU, 2 (24) (in Russ.) (2014)

3. Kundrak J., Hard turning: manufacturing possibilities and economic efficiency, J Equipment and tooling for professionals, 9, pp. 24-27 (in Russ.) (2009)

4. Kundrak J., Gyani K. et al, Hard turning-machining without cooling, High technologies in mechanical engineering, Proceedings of NTU "KPI”, Kharkov, 1 (4), pp. 54-58 (in Russ.) (2001)

5. Goldfarb V. I., Innovative way of development of spiroid gear and gearbox production, Theory and practice of gears and gearbox engineering, Izhevsk: ISTU, pp. 112-114 (in Russ.) (2017)

6. Guyev A. A., Kovalchyuk E. R., Koselov I. M. et al, Technology of mechanical engineering, Moscow: Mashinostroyeniye, p. 406, Fig. 5.32, Fig. 5.33 (in Russ.) (1986)

7. State Standard GOST 18498-89. Worm gears. Terms, definitions and designations (in Russ.).

8. Georgiev A. K., Goldfarb V. I., To the issue of cutting schemes by a cutter and profiles of cylindrical linear worms of spiroid (doubleenveloping worm) gears, Mechanical gears, Izhevsk, pp. 76-86 (in Russ.) (1972)

9. Volkov A. E., Gundayev S. A., Sheveleva G. I., Triangulation algorithms of modeling the generation processes and meshing of gears, J Mashinovedeniye, 6, pp. 60-65 (in Russ.) (1986)

10. Kugultinov S.D., Kovalchyuk A.K., Portnov I.I., Technology of machining structural materials, MGTU im. N.E. Baumana, p. 114 (in Russ.) (2006)

11. Technology of production and methods for providing the quality of gearwheels and gears. Studying guide, eds. V.E. Starzhinsky, M.M. Kane, Saint-Petersburg, Professiya, (in Russ.) (2007)

12. Trubachev E., Loginov S., Bogdanov K. et al, Efficient Schemes and Methods for Gear Machining of Spiroid Gearwheels and Worms, "Advanced Gear Engineering", MMS 51, Springer Int. Publ. AG Switzerland, pp. 465-480, ISBN: 978-3-319-60398-8, DOI 10.1007/978-3-31960399-5 (2018) 